\title{
Ritorno alla bioetica della ricerca (e del sistema che la supporta)
}

\author{
Alberto Edefonti ${ }^{1}$, Giada Albertario ${ }^{1}$, Carlo Agostoni ${ }^{2}$ \\ ${ }^{1}$ UOC Nefrologia e Dialisi Pediatrica, Fondazione Ca' Granda IRCCS, Ospedale Maggiore Policlinico, Milano \\ ${ }^{2}$ UOC Pediatria Media Intensità, Fondazione Ca' Granda IRCCS, Ospedale Maggiore Policlinico, Milano
}

\begin{abstract}
It's time to return to the bioethics of research (and the system supporting it)
The article by Lombardi and the comments by Timio and Floccari underlined the ethical biases of the peer review process and, more generally, of the so-called "industry of scientific evidence".

We further analyze the problematic nature of the peer review mechanisms, both on the Editor's and the Reviewer's side, with the Declarations of Conflict of Interest and Financial Support being the most qualifying proposals for a change.

The diffusion of the "open science" helps to improve the sharing of Author's data with the scientific community. However, the active role of institutions, like FDA and EFSA, in the deep analysis of the results of major articles dealing with drugs and nutrients is highly welcome.

The world of scientific publishing is growing fastly, driven more by the law of economic profits than by the interest of science and patients' care. The average quality of the papers is then decreasing, but this is merely functional to inflate researchers' I.F. and curriculum.

In front of such a critical situation, a couple of bioethical values must be recalled: the first is the responsibility, transparency and honesty of the researcher, which are the result of a process of continuous education and adherence to the principles of the Federazione Italiana delle Società Medico-Scientifiche. A structural reform of the Academic system is also the "conditio sine qua non"; new, ethically based figures of researchers may arise in the next future. The second value is the control of quality and ethics of research, with special focus on the role of the Ethical Committee in filtering methodologically inadequate or financially non transparent study proposals.
\end{abstract}

Keywords: Research bioethics, Open science, Peer review, Financial disclosure, Ethical committee

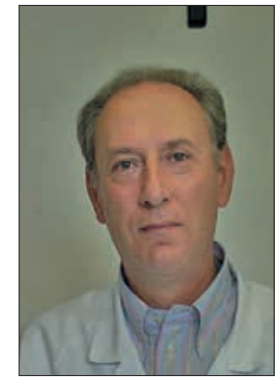

Alberto Edefonti

\section{Premessa}

Siamo stati molto colpiti dall'articolo di Duccio Lombardi "Il progresso umano: quando la scienza non accresce la conoscenza. Il caso delle cellule STAP" (1), dal relativo editoriale di Mario Timio "Ritorno alla bioetica della peer review" (2) e dall'ulteriore commento di Fulvio Floccari “'L'industria dell'evidenza scientifica e la salute del metodo galileiano" (3).

Accepted: August 26, 2015

Published online: September 14, 2015

Indirizzo per la corrispondenza:

Dr Alberto Edefonti

UOC Nefrologia e Dialisi Pediatrica

Fondazione IRCCS Ospedale Maggiore Policlinico Milano

Via Commenda 9, 20122 Milano

alberto.edefonti@policlinico.mi.it

Essi ci propongono, infatti, domande che stanno alla radice del nostro operare come medici e ricercatori. Per questo, abbiamo risposto prontamente all'invito di Marco Lombardi di scrivere un breve commento, più allo scopo di contribuire con il modesto valore dell'esperienza che per seria intenzione di intervenire nei complessi processi, individuali e collettivi, che portano alla pubblicazione di articoli scientifici.

Certamente, il momento che la ricerca sta attraversando è critico e la possibilità che una parte dei dati scientifici pubblicati non fornisca informazioni veritiere è da considerare con attenzione (4). Prenderne piena coscienza è, quindi, fondamentale e impegnarsi a contrastare, ciascuno nella propria realtà, il meccanismo perverso della frode scientifica è, a nostro avviso, essenziale.

A questo proposito, dobbiamo dichiarare, innanzitutto, che condividiamo pienamente l'analisi e le proposte operative di Duccio Lombardi, Mario Timio e Fulvio Floccari: I'idea di aprire la ricerca individuale al giudizio della comunità scientifica (open science), rendendo pubblici e valutabili i dati di "Pazienti e Metodi" e permettendo la riproducibilità dei risul- 
tati, è, sicuramente, una modalità importante per "favorire la scienza, la medicina, la cura e, soprattutto, il paziente".

Inoltre, sviluppare la capacità di "critical appraisal" rende certamente "più liberi dai condizionamenti della letteratura scientifica di scarsa qualità" (3) e permette scelte cliniche consapevoli.

Vorremmo completare queste idee centrali con due commenti riguardanti la peer review e l'open science, per poi passare a considerazioni più a monte, che abbiamo pensato di riassumere nel titolo dell'articolo: "Ritorno alla Bioetica della ricerca (e del sistema che la supporta)".

\section{Commenti sul meccanismo della peer review}

La "revisione tra pari" è un incarico su invito a cui contribuiamo quasi quotidianamente, ma, proprio per questo, meritevole di più profonde riflessioni.

Non dobbiamo dimenticare, infatti, che la scelta dei revisori è generalmente operata dall'Editor o dall'Associate/Section Editor e dovrebbe essere basata sul criterio della competenza, spesso valutata in automatico da un'analisi della letteratura su Pub-MED. Ma è davvero questa una garanzia di obiettività? Certamente, l'Editor si assume una grande responsabilità, non avendo alcun controllo sopra di sé. Ma può accadere che la scelta dei revisori sia basata su criteri più geo-politici che di merito, se non, addirittura, su criteri lobbistici. In alcune riviste prestigiose, inoltre, è l'Editor a filtrare gli articoli meritevoli di una revisione da parte degli esperti, decidendo, quindi, arbitrariamente il rifiuto o meno dell'articolo.

Dipendono sempre dall'Editor e dal Comitato Editoriale le politiche di pubblicazione della rivista, da cui possono nascere i bias rappresentati dagli argomenti di moda del momento e dalla preferenza data agli studi con risultati positivi piuttosto che negativi: entrambi sono utili per aumentare l'impact factor della rivista, ma non sempre contribuiscono a rappresentare l'oggettività della ricerca e la cura del malato.

A volte, inoltre, l'Editor e l'Associate/Section Editor si possono trovare in conflitto, potendo uno dei due trovare un interesse per un tipo di pubblicazione e l'altro vedersi obbligato a contrastarne il parere, sulla base della mancanza di plausibilità biologica o della trasformazione di un outcome primario, non altrimenti giustificate dalla dimensione del campione.

Dopo il versante dell'Editor, due parole anche sul versante del revisore. La richiesta di un eventuale conflitto di interesse del revisore si sta sempre più diffondendo nel processo online di valutazione degli articoli, e questo è, ovviamente, assai positivo sul piano etico. Rimane comunque il fatto che tale dichiarazione dovrebbe diventare obbligatoria, anche se non porrebbe del tutto al riparo dal fenomeno riprovevole del "fare cartello" da parte di gruppi di ricercatori che desiderano mantenere l'esclusiva della pubblicazione in determinati settori.

La dichiarazione del financial support relativo alla ricerca da pubblicare è altrettanto importante sul piano etico e dovrebbe essere sempre richiesta dall'Editor della rivista e specificata in calce all'articolo pubblicato.

Questo criterio di financial disclosure sta divenendo la regola per le presentazioni orali nei più importanti congressi internazionali e viene, inoltre, costantemente richiesto ai revisori prima della valutazione di proposte di ricerca da parte di Fondazioni o istituzioni pubbliche, ai fini del loro finanziamento. Peraltro, il mondo scientifico sa bene che tali disclosure sono puramente pertinenti, nella forma e nella sostanza, all'etica soggettiva del dichiarante e di eventuali terze parti che possono essere a conoscenza di rapporti finanziari in maniera confidenziale.

\section{Commenti sulla diffusione dell'open science}

Riguardo alla diffusione del fenomeno dell'open science, non vi è alcun dubbio che questa andrà incontro a sempre maggiore sviluppo, in ciò favorita dall'evoluzione dell'information technology e della rete web.

Sarebbe stata impensabile, alcuni anni fa, la pubblicazione in cartaceo di tutti i dati relativi a pazienti, metodi e risultati di uno studio clinico sperimentale, cosa che è, invece, oggi possibile sui siti web di case editrici, come addendum dell'articolo stampato.

Un ulteriore esempio di open science è rappresentato da siti come https://clinicaltrials.gov/, dove è possibile "postare" gli studi prima che vengano iniziati, così da evitare doppioni nel campo della ricerca e da favorire, attraverso la lettura critica della metodologia dello studio postato, progetti di ricerca metodologicamente disegnati meglio.

$\mathrm{Ma}$, anche in questi casi, la garanzia della veridicità dei dati o, almeno, dell'aderenza delle conclusioni ai risultati potrà essere opera solamente di quelle istituzioni che, come FDA, EFSA o simili, nel verificare la coerenza dei risultati con la ricerca di claim per farmaci o nutrienti, andranno a confrontare in maniera comparata e inappellabile i risultati stessi.

\section{Ritorno all'etica della ricerca (e del sistema che la supporta)}

È sotto gli occhi di tutti la pletora di riviste medico-scientifiche che compaiono sulla scena, ultimamente soprattutto sotto forma di "open journal". Non passa giorno che non si ricevano inviti a far parte di comitati editoriali e a presentare articoli per riviste di questo tipo: è, evidentemente, un business in crescita, come sottolineato anche da Floccari, spesso gestito in paesi emergenti. Di conseguenza, è in aumento la pubblicazione di articoli il cui valore è discutibile, in quanto inutili, ripetitivi di altre esperienze, mal disegnati e metodologicamente scorretti, dal momento che sfuggono al filtro di un attento processo di revisione.

La rete web rischia, pertanto, di trasformare la propria natura da garanzia di accessibilità e democraticità scientifica ad anarchia e incontrollabilità, in mancanza di un codice chiaro di autoregolamentazione. Tutti siamo stati testimoni del recente intervento di Umberto Eco a Torino e delle polemiche 
suscitate, a torto o a ragione, contro l'assenza di controllo delle testimonianze sulla rete web.

Con il numero delle riviste, aumenta anche la richiesta di peer review e, con essa, il rischio di superficialità, fretta o prevenzione nel giudizio, come si può facilmente riscontrare leggendo le revisioni dei co-reviewer di un medesimo articolo, dopo che è stato espresso dall'Editore il giudizio finale riguardo alla pubblicazione.

\section{La domanda è, quindi: perché si pubblica e a chi è funzionale questo sistema?}

II vantaggio (economico) è sicuramente per il sistema editoriale (cartaceo e/oppure open). Per i ricercatori, la pubblicazione è utile per aumentare il proprio impact factor e favorire la carriera accademica e il prestigio personale e, non da ultimo, l'introito economico. Infine, va citato il vantaggio economico delle aziende farmaceutiche e di presidi medici, di per sé non negativo in origine, ma che può diventare diabolico nella ricerca di un profitto fine a se stesso.

Purtroppo, senza voler eccedere in un facile moralismo, l'eccessiva competitività nel campo della ricerca ("publish or perish") e i conflitti di interesse, scientifici ed economici, trovano facile esca nella natura umana e possono portare, in mancanza di solidi principi morali, a veri e propri casi di disonestà professionale, come quello riportato da Duccio Lombardi (1) e, probabilmente, da molti altri.

In conclusione, è difficile riconoscere oggi che il sistema della ricerca abbia come fini principali l'interesse della persona malata e il progresso delle conoscenze mediche.

Il primo valore bioetico da richiamare è, quindi, quello di "responsabilità, trasparenza e correttezza del ricercatore", che dovrebbero essere inculcate sin dalla fase di formazione, come raccomandato giustamente da Lombardi e Timio, e mantenute nel tempo dall'aderenza ai principi comportamentali del codice della Federazione Italiana delle Società Medico-Scientifiche (5).

Ciò significa, per esempio, resistere, nella pratica scientifica quotidiana, alla tentazione del "piccolo aggiustamento" del dato ai fini di una migliore significatività statistica dei risultati, come anche evitare la "larga via" della facile pubblicazione su riviste, specialmente on-line, di basso profilo per scegliere la "via stretta" della pubblicazione su riviste autorevoli con maggiore garanzia di peer review adeguata. II curriculum scientifico del medico ne risulterà meno "dopato", ma più rispettoso del rigore scientifico e dell'interesse del paziente.

II ricercatore universitario italiano si trova certamente a lavorare all'interno di un sistema le cui regole sono mutate notevolmente negli ultimi anni. La ricerca pediatrica e, ancora di più, quella nefrologica pediatrica hanno sofferto pesantemente per questo cambiamento, tanto che la Società Italiana di Ricerca Pediatrica ha invitato, nei mesi scorsi, i propri soci a rispondere al questionario "L’apoptosi annunciata della ricerca clinica (pediatrica)", ovvero "The vanishing pediatrician scientist", dove sono elencate in 24 punti le principali criticità del sistema italiano della ricerca scientifica.

È chiaro che solo una riforma efficace di questo sistema potrà, nel prossimo futuro, far emergere figure di ricercatori che contemperino la giusta ambizione di successo con il rispetto ben inculcato di regole morali e, soprattutto, con una visione etica della propria funzione professionale.

Il secondo valore bioetico è quello del controllo sulla qualità e sull'eticità della ricerca. Abbiamo molto parlato della rilevanza bioetica della "revisione tra pari", ma un filtro importante per porre un freno alle ricerche inutili o, peggio, prezzolate, metodologicamente inadeguate o di scarso interesse per il malato è quello del Comitato Etico, che ha il compito di primo filtro delle proposte di studio dei ricercatori, con lo scopo precipuo di evitare i fenomeni succitati.

A questo proposito, limitandoci a un commento sui protocolli di ricerca finanziati dall'industria, il loro esame in Comitato Etico dovrebbe essere sempre affiancato da un disclosure form relativo agli interessi economici del ricercatore proponente, a miglior garanzia dell'oggettività dei risultati dello studio.

Un potenziale rischio di valutazione "benevola" di questo tipo di studi potrebbe, però, essere il meccanismo di finanziamento ministeriale di ricerca all'interno degli IRCCS, che dipende dalla produttività scientifica dell'Istituto. Grande attenzione andrebbe, quindi, posta nel tutelare l'interesse del malato, piuttosto che quello dell'Istituzione.

Infine, le riviste scientifiche dovrebbero aprirsi alla pubblicazione di studi con risultati negativi come anche alla valutazione delle repliche degli studi stessi. Solo così si potranno assemblare risultati che portino alla maggiore distanza possibile dall'errore e basati su una numerosità consistente, se non in maniera diretta almeno a livello di analisi e revisioni sistematiche $(4,6)$.

\section{Disclosures}

Financial support: No financial support was received for this submission.

Conflict of interest: The authors have no conflict of interest.

\section{Bibliografia}

1. Lombardi D. II progresso umano: quando la scienza non accresce la conoscenza. II caso delle cellule STAP. Giornale di Tecniche Nefrologiche e Dialitiche. 2015;27(1):42-4.

2. Timio M. Ritorno alla bioetica della "peer review". Editoriale. Giornale di Tecniche Nefrologiche e Dialitiche. 2015;27(1):1-3.

3. Floccari F. L'industria dell'evidenza scientifica e la salute del metodo galileiano. Giornale di Tecniche Nefrologiche e Dialitiche. 2015;27(2):129-30.

4. Ioannidis JP. Implausible results in human nutrition research. BMJ. 2013;347:f6698.

5. Federazione Italiana delle Società Medico-Scientifiche, Ricerca scientifica e sperimentazione clinica 2007.

6. Agostoni C. Primum non nocere. J Pediatr Gastroenterol Nutr Apr. 6, 2015. 